\title{
Chronic venous disease: Literature review
}

\author{
Miguel A. Sierra-Juárez*, José E. Rejón-Cauich, Melvin G. Parada-Guzmán, and \\ Saira A. Castañeda-Morales \\ Service of Vascular Surgery, Hospital General de México “Dr. Eduardo Liceaga”, Mexico City, Mexico
}

\begin{abstract}
The venous system is responsible for bringing blood from the lower limbs to the heart. To achieve this, various mechanisms are activated that work against gravitational force. A very important mechanism is the function of the venous valves. A family history of venous disease is most commonly associated with valve dysfunction, which can lead to venous hypertension, amongst several other risk factors, thus activating a cascade of events characterized by venous dilation and leukocyte migration. Chronic complications can be very expensive in relation to quality of life and the health system. At present, venous disease studies include several diagnostic methods that, together with a wide range of therapeutic tools, have achieved excellent results in the quality of life of patients.
\end{abstract}

Key words: Endovenous thermal ablation. Saphenous vein. Vascular surgery. Venous insufficiency.

\section{Introduction}

Chronic venous disease (CVD) is a common disease with an estimated worldwide prevalence of $83.6 \%$. Varicose veins of the lower extremities are the most common cause of chronic venous insufficiency (CVI) and the most severe form of the disease is venous ulceration. It is estimated that $30-40 \%$ of the adult population has varicose veins and up to $6 \%$ of patients with varicose veins develop ulcers at some time in their lives. Up to $30 \%$ of varicose veins may progress to more severe forms of $\mathrm{CVI}$. Almost $1 \%$ of the general population may develop venous ulceration at some time and the prevalence of open venous ulceration is approximately $0.1-0.3 \%{ }^{1}$. The incidence of varicose veins is estimated to be about $2 \% / y^{2}{ }^{2}{ }^{2}$. Congenital venous valve abnormalities are proposed to be the cause of varicose veins ${ }^{3}$.
According to the Framingham Heart Study, the 2-year incidence of varicose veins in women and men was $2.6 \%$ and $2.0 \%$. CVI can occur as a result of primary and secondary causes $(70 \%$ and $30 \%$ of cases, respectively) 4 .

Venous ulcers can affect at least $1-2 \%$ of the elderly population with a significant burden in terms of quality of life and health-care costs ${ }^{5}$.

\section{Pathophysiology and epidemiology of venous disease}

Thirty genetic loci strongly associated with varicose veins have been identified, with the strongest associations occurring in the intron region CASZ1 ( $r$ 11121615; $p=3.71 \times 10-65$ ), which has been implicated in blood pressure. In addition, mutations in the HFE gene have previously been associated with venous ulceration and
Available online: 19-04-2021

Rev Med Hosp Gen Mex. 2021;84(2):80-86 www.hospitalgeneral.mx 0185-1063/@ 2020 Sociedad Médica del Hospital General de Mexico. Published by Permanyer. This is an open access article under the CC BYNC-ND license (http://creativecommons.org/licenses/by-nc-nd/4.0/) 
thromboembolism ${ }^{6}$. Studies seem to suggest an increase in varicose veins in type I collagen content and a decrease in type III collagen content, the latter being a factor in venous elasticity, both elastin, and laminin content decreases in varicose veins. Laminins are a group of glycoproteins of high molecular mass (140-400 $\mathrm{kDa}$ ) that is part of the basal lamina associated with other proteins such as collagen, entactin, proteoglycans, and fibronectins ${ }^{7}$. The calf muscle pump is the most important pump in providing venous return of the lower extremity, but the quadriceps and hamstring muscle pumps in the thigh are of importance in pumping blood to the heart ${ }^{8}$.

Recently in a study by Rusinovich, the C6 CEAP classification (Table 1) compared to $\mathrm{C} 2$ was associated with higher atrial contribution to right ventricular filling, higher atrial contraction, and higher atrial ejection force. Clinical class C6 CEAP was associated with impaired relaxation or diastolic dysfunction of the right heart ${ }^{9}$. The Vein Consult program is an international, observational, prospective survey to collect global epidemiological data. It reports on $\mathrm{CVI}$; it estimates the global prevalence of $\mathrm{CVI}$ at $83.6 \%(\mathrm{C} 0-\mathrm{C} 6)$ with a global prevalence of $\mathrm{C} 1$ to $\mathrm{C} 6$ at $63.9 \%^{10}$. In a meta-analysis, it was observed that $4677(35.0 \%)$ of 13,361 women with parity $>1$ developed varicose veins. In the control group with 3746 women with no history of pregnancy, only 782 $(20.9 \%)$ cases of varicose veins were reported. Progesterone inhibits smooth muscle contraction, while estrogen causes vasodilatation. Both mechanisms may result in venous insufficiency caused by increased capacity and dilatation of the venous system, together with venous outflow obstruction by the pregnant uterus and increased weight gain during pregnancy. More research is needed to explain the association between pregnancy and venous insufficiency. Pregnancy increases the risk of developing varicose veins (odds ratio, 1.82; $95 \%$ confidence intervals [Cl], 1.43-2.33) 1.82-fold ${ }^{11}$. Venous outflow obstruction affecting the iliocaval segment has been identified in $10 \%-30 \%$ and is therefore of paramount importance in deep system analysis of $\mathrm{CVI}^{12}$. A prevalence of both deep (1.7\%) and superficial (2.2\%) vein thrombosis of the venous systems was reported in patients with CVI undergoing ultrasound scans ${ }^{13}$.

\section{Health costs involved in the care of patients with venous disease}

In the United States, it has been estimated that the direct medical costs of CVD range between $\$ 150$
Table 1. The 2020 update of the CEAP classification system and reporting standards

\begin{tabular}{|l|l|}
\hline \multicolumn{1}{|c|}{$\begin{array}{l}\text { Summary of clinical } \\
\text { C class description }\end{array}$} \\
\hline C0 & $\begin{array}{l}\text { No visible or palpable signs } \\
\text { venous disease }\end{array}$ \\
\hline C1 & $\begin{array}{l}\text { Telangiectasia or reticular } \\
\text { veins }\end{array}$ \\
\hline C2 & Varicose veins \\
\hline C2r & Recurrent varicose veins \\
\hline C3 & Edema \\
\hline C4 & $\begin{array}{l}\text { Changes in skin and } \\
\text { subcutaneous tissue } \\
\text { secondary to CVD }\end{array}$ \\
\hline C4a & Pigmentation or eczema \\
\hline C4b & $\begin{array}{l}\text { Lipodermatosclerosis or } \\
\text { atrophie blanche }\end{array}$ \\
\hline C4c & Corona phlebectatica \\
\hline C5 & Healed \\
\hline C6 & Active venous ulcer \\
\hline C6r & Recurrent active venous ulcer \\
\hline
\end{tabular}

\begin{tabular}{|c|c|}
\hline $\begin{array}{c}\text { Summary of etiologic (E) } \\
\text { classification }\end{array}$ & $\begin{array}{c}\text { Summary of } \\
\text { pathophysiologic (P) }\end{array}$ \\
\hline E class Description & P class Description \\
\hline
\end{tabular}

\begin{tabular}{|l|l|l|l|}
\hline Ep & Primary & $\operatorname{Pr}$ & Reflux \\
\hline Es & Secondary & Po & Obstruction \\
\hline Esi & Secondary - intravenous & $\begin{array}{l}\mathrm{Pr} \\
0\end{array}$ & $\begin{array}{l}\text { Reflux and } \\
\text { obstruction }\end{array}$ \\
\hline Ese & Secondary - extravenous & $\mathrm{Pn}$ & $\begin{array}{l}\text { No pathophysiology } \\
\text { identified }\end{array}$ \\
\hline Ec & Congenital & & \\
\hline En & No cause identified & \\
\hline
\end{tabular}

\begin{tabular}{c} 
Summary of anatomic (A) classification \\
\hline A Class Description
\end{tabular}

As Superficial

Old New* Description

1. Tel Telangiectasia

1. Ret Reticular veins

2. GSVa Great saphenous vein above knee

3. GSVb Great saphenous vein below knee

4. SSV Small saphenous vein 
Table 1. The 2020 update of the CEAP classification system and reporting standards

\begin{tabular}{|l|l|}
\hline \multicolumn{1}{|c|}{ Summary of anatomic (A) classification } \\
\hline AASV Class Description \\
\hline 5. NSV Non-saphenous vein \\
\hline Ad & Deep \\
\hline Old New* Description \\
\hline 6. IVC Inferior vena cava \\
\hline 7. CIV Common iliac vein \\
\hline 8. IIV Internal iliac vein \\
\hline 9. EIV External iliac vein \\
\hline 10. PELV Pelvic veins \\
\hline 11. CFV Common femoral vein \\
\hline 12. DFV Deep femoral vein \\
\hline No venous anatomic location identified \\
\hline 13. FV Femoral vein \\
\hline Perforator \\
\hline Old New* Description \\
\hline 17. TPV Thigh perforator vein \\
\hline 15. TIBV Crural (tibial) vein \\
\hline 15. PRV Peroneal vein \\
\hline 15. ATV Anterior tibial vein \\
\hline 15. PTV Posterior tibial vein \\
\hline 16. MUSV Muscular veins \\
\hline 16. GAV Gastrocnemius vein \\
\hline 16. SOV Soleal vein \\
\hline An \\
\hline A
\end{tabular}

CVD: chronic venous disease

*New specific anatomic location (s) to be reported under each

$\mathrm{P}$ (pathophysiologic) class to identify anatomic location (s) corresponding to $P$ class.

million and \$1 billion annually. In the United Kingdom, $2 \%$ of the national health budget per year (USD 1 billion) is spent on leg ulcer management. The cost of caring for patients with CVI is estimated at EUR 600900 million in Western Europe, representing $2 \%$ of health-care expenditure. The estimated average direct cost for treating each ulcer is EUR 9000, representing $90 \%$ of the total expenditure for CVI patients ${ }^{14}$.

\section{Venous disease clinical diagnosis and laboratory study}

In a study on biomarkers regarding CVI by Mosmiller, it was observed that the neutrophil count and neutrophil/lymphocyte ratio were significantly higher in the severe-to-mild CVI group. Moreover, the neutrophil/ lymphocyte ratio can serve as an independent predictor of severity of CVI when it is $>2.91$ with $74 \%$ sensitivity and $77 \%$ specificity ${ }^{15}$.

Clinical assessment is found in CVD-related signs and symptoms. Unfortunately, they are non-specific and difficult to associate with venous disease. Symptoms associated with CVD include leg heaviness, aching and throbbing, tiredness, fatigue, itching, tingling or burning sensation, and nocturnal cramps ${ }^{16}$. At present, research in patients with superficial venous insufficiency is invariably limited to assessment of the presence and sites of reflux. Flow volume measured by plethysmography may be more representative as indicated in a recent publication on venous reflux quantification. However, the anterograde saphenous volume has been shown to be a determinant of the resulting reflux volume. Thus, the recirculation index may be an improvement in venous reflux quantification ${ }^{17}$.

The anatomical and functional assessment of the venous system should be performed by Doppler ultrasound, the ideal method, as it is reproducible and non-invasive. A Doppler flow duration of $>500$ ms with a diameter of $>3.5 \mathrm{~mm}$ was considered as pathological perforators. Color Doppler shows a sensitivity of $80 \%$ with an accuracy of $10 \%$ and the desired $\mathrm{Cl}$ of $95 \%{ }^{3}$.

Venous reflux has traditionally been managed first. However, advances in diagnostic and imaging techniques, mainly intra-vascular ultrasound, have allowed us to understand better the obstructive physiology of venous disease. In fact, venous outflow obstructions affecting the iliocaval segment have been identified in $10-30 \%$ of patients with severe venous insufficiency ${ }^{18}$. By means of diagnostic equipment, $v$

enous disease analysis is more specific to differentiate the affected segments to be treated.

\section{Venous healthcare and medical treatment of venous disease}

The Society for Vascular Surgery (SVS) and the American Venous Forum have developed clinical practice guidelines for the care of patients with varicose veins of the lower limbs. They follow compression therapy for patients with symptomatic varicose veins 
(GRADE 2C) but recommend against compression therapy as primary treatment in patients who are candidates for saphenous vein ablation (GRADE 1B). We recommend compression therapy as primary treatment to aid healing of venous ulceration (GRADE 1B). To decrease the recurrence of venous ulceration, we recommend ablation of incompetent superficial veins in addition to compression therapy (GRADE $1 A)^{19}$. In one study, we observed the change in ankle joint range of motion and muscle strength values measured with an isokinetic dynamometer, pain scores, quality of life scale, and venous return time in patients with CVI. In conclusion, it has been determined that increased muscle strength affects the venous pump and this improvement ensures venous function ${ }^{20}$. Compression is not recommended in patients with $\mathrm{ABI}<0.5^{21}$.

Venoactive drugs are deemed an important component of the medical (conservative) treatment of CVD. According to available evidence, four drugs (MPFF, hydroxyethylrutosides, ruscus extract, and diosmin) are able to act in reducing edema. The first three showed significant reduction compared to placebo whereas diosmin did not. MPFF was significantly superior to hydroxyethylrutosides and ruscus extract ${ }^{5,22}$. Several landmark studies have demonstrated the cascade of interactions that correlate with increased venous pressure and capillary perfusion: white blood cell adhesion and migration, endothelial leukocyte activation, capillary permeability, increased vascular proliferation and altered lymph flow, leukocyte trapping, and skin pathology. Elevated L-selectin during venous hypertension was considered an indication that leukocyte binding occurred. A systematic literature review focused on the use of micronized purified flavonoid fraction (MPFF) in the treatment of CVD. An overall level of evidence supports the recommendation of the therapeutic use of MPFF with beneficial outcomes without serious adverse events ${ }^{23}$. MPFF significantly improved nine defined leg symptoms, including pain, heaviness, swelling, cramps, paraesthesia, burning sensation, and pruritus (itching). MPFF is highly effective in improving leg symptoms, edema, and quality of life in patients with CVD $^{24}$.

Sulodexide protects the endothelium by restoring endothelial glycocalyx, preventing venous endothelial cell apoptosis and inhibiting endothelial cell release of reactive oxygen species and pro-inflammatory chemokines and interleukins (IL), such as monocyte chemotactic protein and IL-625. Sulodexide has a venoactive effect on the main signs and symptoms of venous disease, such as pain, cramps, heaviness, and edema without increasing the risk of adverse effects. It is also likely to exert a systemic effect on the course of CVD by interfering with inflammatory chemokines ${ }^{26}$.

There are undoubtedly multiple pharmacological options, and every day new properties are discovered that improve the consequences caused by venous hypertension in patients with CVD.

\section{Surgical treatment of CVD}

Surgical treatment of varicose veins has two objectives. On the one hand, it is aimed at correcting the problem that caused the varicose veins. On the other hand, the aim is to eliminate the visible veins that have become dilated. Nowadays, there are different techniques for varicose vein surgery. High ligation with vein stripping (open surgery) involves the closure of the femoral saphenous junction and its branches through several incisions. Thermal ablation is a catheter-assisted procedure that uses radiofrequency or laser energy causing the vein to collapse. Foam sclerotherapy for large veins makes it possible to close and seal the vein. Endoscopic surgery by means of camera-guided trocars for perforator vein clamping and finally non-thermal and non-tumescent methods (mechanochemical ablation and cyanoacrylate glue) (Fig. 1).

In response to less invasive treatment with endovenous treatment, radiofrequency ablation (RFA) and endovenous laser ablation (EVLA) ${ }^{27}$ have been developed. Endovenous thermal ablation (EVTA) in a meta-analysis review showed that technical success rates were $84.8 \%$ for EVLA, $88.7 \%$ for RFA, and $32.8 \%$ for ultrasound-guided foam sclerotherapy (UGFS). In conclusion, both EVLA and RFA are effective in long-term great saphenous vein occlusion ${ }^{28}$. EVTA with EVLA or RFA is safe and effective for the treatment of recurrent varicose veins resulting from residual insufficiency of greater saphenous vein surgical treatment. RFA is superior to $980 \mathrm{~nm}$ EVLA in terms of post-procedure ecchymosis ${ }^{29}$. Endothermal ablation is an effective treatment for $\mathrm{CVI}$ and most patients remain free of reflux. Recanalization of an isolated venous segment after RFA, although shown in recent literature to be affected by anatomical risks, appears to be a sporadic phenomenon with respect to clinical risk factors ${ }^{30}$. Laser treatments must sometimes be accompanied by other therapeutic options, although obliteration of the GSV above the knee improves symptoms independently of persistent below-knee reflux, the latter appears to be responsible for residual symptoms and a greater need for sclerotherapy for residual varicose veins ${ }^{31}$. 


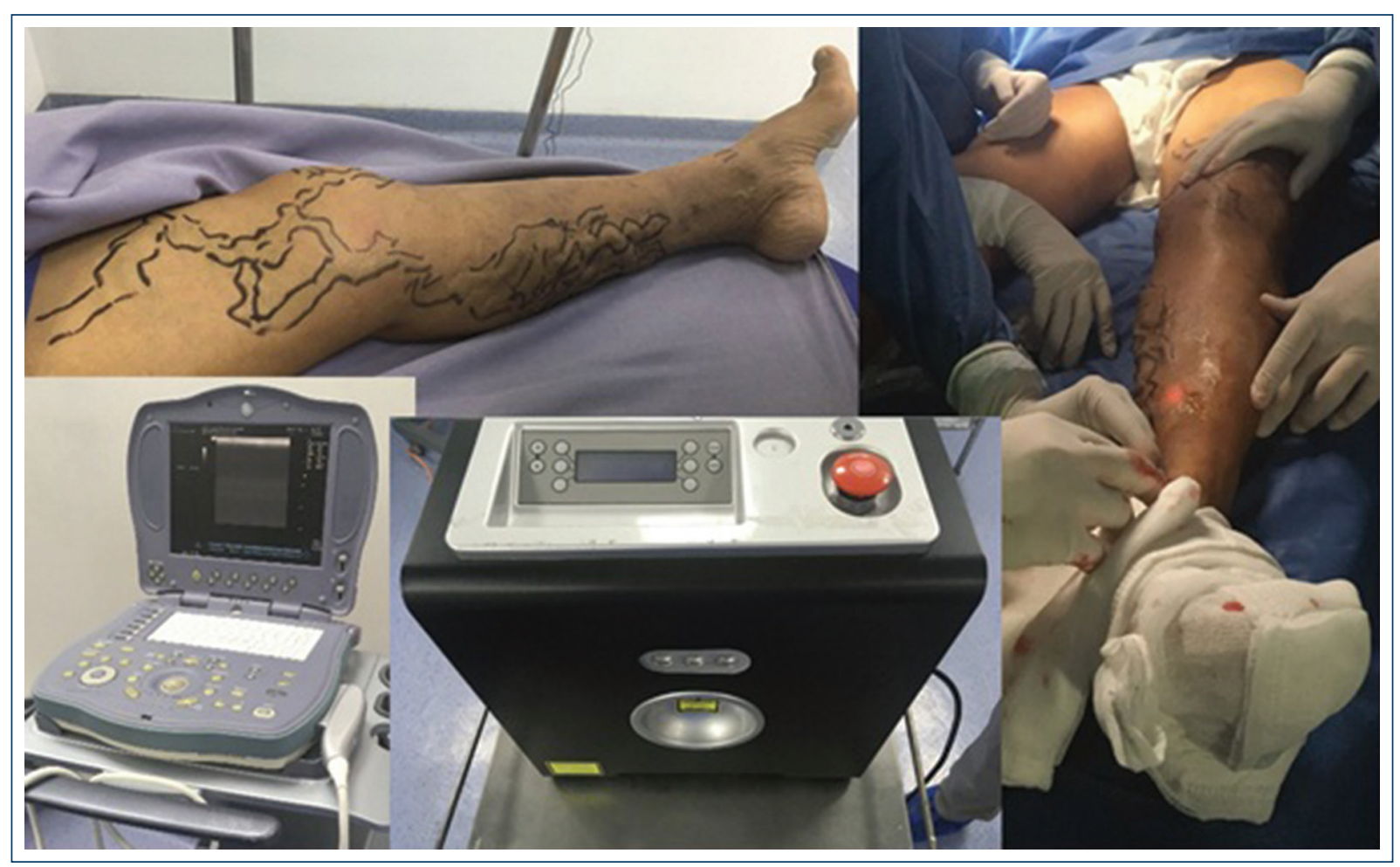

Figure 1. Surgical ultrasound marking and endovenous $1470 \mathrm{~nm}$ laser ablation with radial fiber for chronic venous disease.

Regarding the long-term effectiveness of thermal ablation of GSVs by radiofrequency (RPSA) or EVLA with less traumatic radial tip fibers (RTF), we have concluded that both procedures have an equally high long-term GSV obliteration rate and the treatments are equally clinically effective ${ }^{32}$. Thermal ablation treatments have the highest incidence of endothermic heat-induced thrombosis (EHIT) after EVTA with RFA than with EVLT. However, the overall incidence of EHIT is relatively low $^{33}$. The incidence of thrombotic complications after EVTA of varicose veins is uncertain. Similar results were found when the RFA and EVLA groups were analyzed separately ${ }^{34}$. In a 20 -centre trial, early endovenous ablation resulted in faster healing of venous ulcers and more ulcer-free time than delayed endovenous ablation ${ }^{35}$.

In conclusion, EVLA/RFA should be preferred to open surgery and foam sclerotherapy in the treatment of venous incompetence ${ }^{36}$.

As for the surgical technique of foam sclerotherapy for great saphenous vein treatment, the results are inferior to surgery to eliminate venous reflux but the advantage in some studies is that patients returned to daily activities more quickly. Vein diameter greater than $6 \mathrm{~mm}$ had worse closure results than those with a diameter of five or less. Patients undergoing ultrasound-guided sclerotherapy (UGS) have better quality of life than surgical patients do, after 4 weeks of treatment due to less pain. In the literature, most studies show reduced evidence due to selection and randomization bias. Long-term results are still lacking and need to be controlled by randomized trials ${ }^{1,37}$. We compared the effectiveness of EVLA, RFA, and UGFS versus conventional surgery in the treatment of varicose veins. UGFS effectiveness compared to conventional surgery in the treatment of small saphenous varicose veins (SSV) is uncertain ${ }^{38}$.

A third treatment option, first described by Hauer in the 1980s, subfascial endoscopic perforator surgery (SEPS), has been performed to treat incompetent perforator veins in cases with advanced skin changes ${ }^{39}$. SEPS, which is performed for perforator ligation, requires endoscopic installation and expertise. The most commonly performed operation to address perforator incompetence is still open subfascial ligation or puncture phlebotomy ${ }^{3}$.

Finally, there are cyanoacrylate adhesive devices (CAC) for saphenous vein closure or devices that inject foam into the saphenous vein while rotating at high speed inside it. The combination of the chemical effect of the foam with 
the mechanical effect of the catheter rotation maximizes its effect and makes it faster. The 36-month follow-up of the first human use of cyanoacrylate adhesive for the treatment of saphenous vein incompetence was $94.7 \%$. Intravenous polidocanol foam (Varithena; sclerosing agent) demonstrated a 1-year occlusion rate of $73 \%{ }^{40}$. A study comparing early and 2-year outcomes for N-butyl cyanoacrylate, EVLA in the treatment of varicose veins, concluded that no difference in occlusion rates was observed in the three modalities, but superior NBC appeared with respect to pain during the procedure, return to work, and decreased pre-operative venous clinical severity scores ${ }^{41}$. In a 12-month follow-up with (CAC) and radiofrequency (RFA), almost identical occlusion rates were demonstrated in the target veins $(96.8 \%$ in the CAC group and $95.9 \%$ in the RFA group). However, the time to complete occlusion was shorter and recanalization was greater with CAC than with RFA ${ }^{42}$. By month 24 , closure rates for CAC and RFA were also equivalent $(95.3 \%$ and $94.0 \%$, respectively) and the recanalization-free rate remained higher in the CAC group, demonstrating the continued non-inferiority of CAC to RFA ${ }^{43}$. In the WAVES study, 1-year results demonstrated the safety and efficacy of CAC for the treatment of GSVs up to $20 \mathrm{~mm}$ in diameter, small saphenous veins (SSVs) and/or accessory saphenous veins, with a $98 \%$ occlusion rate in all veins ${ }^{44}$. CAC does not produce significant thrombosis because the vein walls adhere to the adhesive immediately through the application of external compression resulting in an inflammatory and eventual fibrotic reaction rather than a thrombotic one ${ }^{45}$. The VenaSeal system (non-thermal, non-tumescent, and non-sclerosing technologies) is a promising therapeutic option for anatomic success at 6 months, with fewer adverse effects in patients with venous insufficiency compared to other interventions ${ }^{46}$. Initial 3-months results from the VeClose trial reported non-inferiority of cyanoacrylate endovenous closure (CAC) to RFA with saphenous vein closure rates greater than $99 \%$ for the CAC group and $96 \%$ for the RFA group ${ }^{47}$. Regarding mechanochemical modalities, initial data showed successful GSV occlusion rates with the ClariVein device (through a mechanochemical ablation catheter) at $94 \%$ comparable to RFA (thermal ablation) ${ }^{15,48}$.

This latest mechanochemical alternative seems very promising, but time is required to appreciate all its advantages, but its benefits are supported by the non-use of heat, which is associated with low pain rates, a quick return to normal activities and a better quality of life. What is certain is that every day, there is more and more technology available to treat patients that allow them a quicker return to their daily activities.

\section{Conclusions}

The treatment of CVD is now more specific, thanks to diagnostic methods, as they help us to better identify diseased segments, thus allowing for targeted surgery with the construction of a surgical map. We can assess blood flow within the vein lumen and venous reflux analysis, measure vascular structures and detect complications in the deep venous system, confirm that it is indeed a primary disease and rule out secondary causes. They are a fundamental part of surgery and allow for post-operative vigilance. This technology has been extended to surgical tools that are now minimally invasive with early recovery and are more accessible to patients with higher risk conditions. In addition, they ensure greater safety measures and provide better results such as less postoperative pain as well as better quality of life, with early recovery of the patient to their daily activities.

\section{Conflicts of interest}

The author declares have not any conflicts of interest.

\section{Ethical disclosures}

Protection of human and animal subjects. The authors declare that no experiments were performed on humans or animals for this study.

Confidentiality of data. The authors declare that they have followed the protocols of their work center on the publication of patient data.

Right to privacy and informed consent. The authors have obtained the written informed consent of the patients or subjects mentioned in the article. The corresponding author is in possession of this document.

\section{References}

1. Rabe E, Guex JJ, Puskas A, Scuderi A, Quesada FF, VCP Coordinators Epidemiology of chronic venous disorders in geographically diverse populations: results from the vein consult program. Int Angiol. 2012;31:105-15.

2. Rabe E, Berboth G, Pannier F. Epidemiology of chronic venous diseases. Wien Med Wochenschr. 2016;166:260-3.

3. Sureshkumar S, Vignesh N, Venkatachalam J, Vijayakumar C, Sudharsanan S. Clinical tests combined with color doppler versus color doppler alone in identifying incompetent perforator veins of the lower limb: a prospective analytical study. Cures. 2018;1:e2026.

4. Erick U, Kartal A, Ferhatoglu M. Association between hemorrhoids and lower extremity chronic venous insufficiency. Cureus. 2019;11:e4502.

5. Attaran R, Chaar $\mathrm{Cl}$. Compression therapy for venous disease. Phlebology. 2017;32:81-8.

6. Fukaya E, Flores A, Lindholm D, Gustafsson S, Zanetti D, Ingelsson E, et al. Clinical and genetic determinants of varicose veins. Circulation. 2018;138:2869-80.

7. Attaran R. Latest innovations in the treatment of venous disease. J Clin Med. 2018:7:77 
8. Cetin C, Serbest M, Ercan S, Yavuz T, Erdogan A. An evaluation of the lower extremity muscle strength of patients with chronic venous insufficiency. Phlebology. 2016;31:203-8.

9. Rusinovich $Y$, Rusinovich V. Association between right heart diastolic function and clinical presentation of chronic venous disease and primary varicose veins of lower extremities. Phlebology. 2020;35:513-9.

10. Javier J, Ortiz P. Treatment of chronic venous insufficiency in Latin America. J Vasc Surg Venous Lymphat Disord. 2020;8:667-75.

11. Ismail L, Normahani P, Standfield N. A systematic review and meta-analysis of the risk for development of varicose veins in women with a history of pregnancy. J Vasc Surg Venous and Lym Dis. 2016;4:518-24.

12. Abou Ali AN, Avgerinos E, Chaer RA. Role of venous stenting for iliofemoral and vena cava venous obstruction. Surg Clin North Am. 2018, 98:361-71.

13. Panpikoon T, Wedsart B, Treesit T. Duplex ultrasound findings and clinical classification of lower extremity chronic venous insufficiency in a Thai population. J Vasc Surg Venous and Lym Dis. 2018;7:349-55

14. Gloviczki P, Comerota A, Dalsing M, Eklof BG, Gillespie DL, Gloviczki ML et al. The care patients with varicose veins and associated chronic venous diseases: clinical practice guidelines of the society for vascular surgery and the American venous forum. J Vasc Surg. 2011;53:2S-48S.

15. Mosmiller L, Steele K, Shrader C, Petrone AB. Evaluation of inflammatory cell biomarkers in chronic venous insufficiency. Phlebology. 2017;32:634-40.

16. Carman T, Omari AA. Evaluation and management of chronic venous disease using the foundation of CEAP. Curr Cardiol Rep. 2019;21:114.

17. Lattimer C, Rudolphi PB, Recke A, Geroulakos G, Kalodiki E, Kahle BK. Comparison of four haemodynamic tests that quantify superficial venous insufficiency. Eur J Vasc Endovasc Surg. 2019;57:570-7.

18. Abou Ali AN, Avgerinos E, Chaer R. Role of venous stenting for iliofemoral and vena cava venous obstruction. Surg Clin North Am. 2018;98:361-71.

19. Wittens $C$, Davies AH, Baekgaard N, Broholm R, Cavezzi A, Chastanet S, et al. editor's choice management of chronic venous disease: clinical practice guidelines of the european society for vascular surgery (ESVS) Eur J Vasc Endovasc Surg. 2015;49:678-737.

20. Ercan S, Cetin C, Yavuz T, Demir HM, Atalay YB. Effects of isokinetic calf muscle exercise program on muscle strength and venous function in patients with chronic venous insufficiency. Phlebology. 2018;33:261-6.

21. Dahm K, Myrhaug H, Strømme H, Fure B, Brurberg KG. Effects of preventive use of compression stockings for elderly with chronic venous insufficiency and swollen legs: a systematic review and meta-analysis. BMC Geriatr. 2019;19:76.

22. Guo L, Huang R, Zhao D, Xu G, Liu H, Yang J, et al. Long-term efficacy of different procedures for treatment of varicose veins. A network meta-analysis. Medicine. 2019;98:e14495.

23. Bush R, Comerota A, Meissner M, Raffetto JD, Hahn SR, Freeman K Recommendations for the medical management of chronic venous disease: the role of micronized purified flavanoid fraction (MPFF). Phebology 2017;32:3-19.

24. Kakos K, Nicolaides N. Efficacy of micronized purified flavonoid fraction (Daflon $\AA$ ) on improving individual symptoms, signs and quality of life in patients with chronic venous disease: a systematic review and meta-analysis of randomized double-blind placebo-controlled trials. Int Angiol. 2018;37:143-54.

25. Flota CL, Frati MA, Velázquez HA. Chronic venous disease treated with sulodexide: a survey among primary care physicians in Mexico. Int Angiol. 2017;36:558-64.

26. Bignamini A, Matuska J. Sulodexide for the symptoms and signs of chronicvenous disease: a systematic review and meta-analysis. Adv Ther. 2020;37:1013-33.

27. Berlramman A, Bootun R, Lane T, Davies AH. Endovenous management of varicose veins. Angiology. 2019;70:388-96

28. Balint R, Farics A, Parti K, Vizsy L, Batorfi J, Menyhei G, et al. Which endovenous ablation method does offer a better long-term technical success in the treatment of the incompetent great saphenous vein? Review. Vascular. 2016;24:649-57.

29. Hwang J, Park S, Soo I. Endovenous thermal ablation of recurrent varicose veins due to residual great saphenous venous insufficiency after saphenous venous surgery: a comparative study. Dermatol Surg. 2018;44:1287-94.
30. Bunnell AP, Zaidi S, Eidson $\mathrm{JL} 3^{\text {rd }}$, Bohannon WT, Atkins MD Jr. Bush RL. Factors associated with saphenous vein recanalization after endothermal ablation. Ann Vasc Surg. 2015;29:322-7.

31. Theivacumar NS, Darwood RJ, Dellagrammaticas D, Mavor Al, Gough MJ. The clinical significance of below-knee great saphenous vein reflux following endovenous laser ablation of above-knee great saphenous vein. Phlebology. 2009;24:17-20.

32. Lawson J, Gauw SA, Vlijmen CV, Pronk P, Gaastra MT, Tangelder MJ, et al. Prospective comparative cohort study evaluating incompetent great saphenous vein closure using radiofrequency powered segmental ablation or $1470 \mathrm{~nm}$ endovenous laser ablation with radial tip fibers (varico 2 sutydy). J Vasc Surg Venous Lymphat Disord. 2018;6:31-40.

33. Chait J, Kibrik P, Alsheekh A, Ostrozhynskyy Y, Marks N, Rajaee S, et al. Radiofrequency ablation increases the incidence of endothermal heat-induced thrombosis. J Vasc Surg. 2020;62:263-7.

34. Healy DA, Kimura S, Power D, Elhaj A, Abdeldaim Y, Cross KS, et al. A systematic review and meta-analysis of thrombotic events following endovenous thermal ablation of the great saphenous vein. Eur $\mathrm{J}$ Endovasc Surg. 2018;56:410-24

35. Gohel M, Heatley F, Liu X, Bradbury A, Bulbulia R, Cullum N, et al. A randomized trial of early endovenous ablation in venous ulceration. $\mathrm{N}$ Engl J Med. 2018;378:2105-14.

36. Boersma D, Kornmann VN, van Eekeren RR, Tromp E, Ünlü C, Reijnen MM, et al. Treatment modalities for small saphenous vein insufficiency: systematic review and meta-analysis. J Endovasc Ther. 2016;23:199-211.

37. de-Abreu GC, de Camargo Júnior O, de-Abreu MF, Braga de-Aquino JL. Ultrasound-guided foam sclerotherapy for severe chronic venous insufficiency. Rev Col Bras. 2017:44:511-20.

38. Vikram SC, Horne M, Dodd PD. Endovenous ablation therapy (laser or radiofrequency) or foam sclerotherapy versus conventional surgical repair for short saphenous varicose veins. Cochrane Database Syst. 2016;29:1-28.

39. Kusagawa $\mathrm{H}$, Haruta N, Shinhara R. Surgical methods and clinical results of subfascial endoscopic perforator surgery in Japan. Phlebology. 2018;33:678-86

40. Almeida JI, Javier JJ, Mackay EG, Bautista C, Cher DJ, Proebstle TM. Thirty-sixth-month follow-up of first-in-human use of cyanoacrylate adhesive for treatment of saphenous vein incompetence. J Vasc Surg Venous Lymphat Disord. 2017;5:658-66.

41. Eroglu E, Yasmin A. A radomised clinical trial comparing N-Butyl cyanoacrylate. Radiofrequency ablation and endovenous laser ablation for the treatment of superficial venous incompetence: two year follow up results. Eur J Vasc Endovasc Surg. 2018:56:553-60.

42. Morrison N, Gibson K, Vasquez M, Weiss R, Cher D, Madsen M, et al. VeClose trial12-month outcomes of cyanoacrylate closure versus radiofrequency ablation for incompetent great saphenous veins. J Vasc Surg Venous Lymphat Disord. 2017:5:321-30.

43. Gibson K, Morrison N, Kolluri R, Vasquez M, Weiss R, Cher D, et al. Twenty-four results from a randomized trial of cyanoacrylate closure versus radiofrequency ablation for the treatment of incompetent great saphenous veins. J Vasc Surg Venous Lymphat Disord 2018;6:606-13.

44. Gibson K, Minjarez R, Gunderson K, Ferris B. Need for adjunctive procedures following cyanoacrylate closure of incompetent great, small and accessory saphenous veins without the use of postprocedure compression: three-month data from a postmarket evaluation of the VenaSeal system (The WAVES study). Phlebology. 2019;34:231-7.

45. Almeida JI, Min RJ, Raabe R, McLean DJ, Madsen M. Cyanoacrylate adhesive for the closure of truncal veins: 60-day swine model results. Vasc Endovasc Surg. 2011;45:631-5.

46. Kolluri R, Chung J, Kim S, Nath N, Bhalla BB, Jain T, et al. Network meta-analysis to compare VenaSeal with other superficial venous therapies for chronic venous insufficiency. J Vasc Surg Venous and Lym Dis. 2020;8:472-81.

47. Morrison N, Gibson K, McEnroe S, Goldman M, King T, Weiss R, et al. Randomized trial comparing cyanoacrylate embolization and radiofrequency ablation for incompetent great saphenous veins (VeClose). J Vasc Surg 2015;61:985-94.

48. Kheirelseid E, Crowe G, Sehgal R. Systematic review and meta-analysis of randomized controlled trials evaluating long term outcomes of endovenous management of lower extremity varicose veins. J Vasc Surg Venous Lymphat Disord. 2018;6:256-70. 